\title{
Reabilitação com prótese fixa metalocerâmica: Acompanhamento de 12 anos
}

\author{
Rehabilitation with prosthesis metal ceramic fixed: 12-year follow-up \\ Rehabilitación con prótesis fija metalcerámica: Seguimiento a 12 años
}

Recebido: 15/06/2021 | Revisado: 24/06/2021 | Aceito: 27/06/2021 | Publicado: 12/07/2021

\author{
Victor Augusto Alves Bento \\ ORCID: https://orcid.org/0000-0003-0558-4239 \\ Universidade Estadual Paulista Júlio de Mesquita Filho, Brasil \\ E-mail: vtrbento97@gmail.com \\ Karolina Braga Costa \\ ORCID: https://orcid.org/0000-0001-8351-6456 \\ Universidade Federal de Mato Grosso do Sul, Brasil \\ E-mail: karolinabraga_@hotmail.com \\ Daisilene Baena Castillo \\ ORCID: https://orcid.org/0000-0003-4261-7503 \\ Universidade Federal de Mato Grosso do Sul, Brasil \\ E-mail: daisilene@hotmail.com
}

\begin{abstract}
Resumo
As próteses parciais fixas metalocerâmicas (PPF) têm sido uma opção de tratamento amplamente utilizadas pelos cirurgiões-dentistas, apresentando taxas de sucesso significativamente altas ao longo prazo. Entretanto, suas taxas são baseadas em ensaios clínicos conduzidos em condições padronizadas e não representam as realidades clínicas. Desta maneira, este trabalho tem como objetivo enfatizar a sobrevida das metalocerâmicas, através de um caso clínico de reabilitação, utilizando liga metálica $\mathrm{Ni}-\mathrm{Cr}$ e cimento de ionômero de vidro modificado por resina com acompanhamento de 12 anos. O caso foi reabilitado com duas PPF posteriores de cinco elementos com dois pônticos e cincos próteses fixas unitárias anterior para o arco superior e duas PPF posteriores de cinco elementos com dois pônticos para o arco inferior. Concluiu-se que as PFF apresentam alta taxa de sucesso há um longo período de acompanhamento, sendo sua longevidade vinculada ao correto planejamento, escolha dos materiais e técnicas.
\end{abstract}

Palavras-chave: Reabilitação bucal; Prótese dentária; Ligas metalo-cerâmicas.

\begin{abstract}
Metal-ceramic fixed partial dentures (PPF) have been a treatment option widely used by dentists, with significantly high long-term success rates. However, their rates are based on clinical trials conducted under standardized conditions and do not represent clinical realities. Thus, this work aims to emphasize the survival of metalloceramics, through a clinical case of rehabilitation, using Ni-Cr metal alloy and resin-modified glass ionomer cement with a 12-year follow-up. The case was rehabilitated with two posterior five-element PPF with two pontics and five anterior unitary fixed prostheses for the upper arch and two posterior five-element PPF with two pontics for the lower arch. It was concluded that the PFF have a high success rate for a long period of follow-up, and their longevity is linked to the correct planning, choice of materials and techniques.
\end{abstract}

Keywords: Oral rehabilitation; Dental prosthesis; Metallo-ceramic alloys.

\section{Resumen}

Las dentaduras postizas parciales fijas (PPF) de metal-cerámica han sido una opción de tratamiento ampliamente utilizada por los dentistas, con tasas de éxito a largo plazo significativamente altas. Sin embargo, sus tasas se basan en ensayos clínicos realizados en condiciones estandarizadas y no representan realidades clínicas. Así, este trabajo tiene como objetivo enfatizar la supervivencia de las metalocerámicas, a través de un caso clínico de rehabilitación, utilizando aleación de metal Ni-Cr y cemento de ionómero de vidrio modificado con resina con un seguimiento de 12 años. El caso fue rehabilitado con dos PPF posteriores de cinco elementos con dos pónticos y cinco prótesis fijas unitarias anteriores para el arco superior y dos PPF posteriores de cinco elementos con dos pónticos para el arco inferior. Se concluyó que los PFF tienen una alta tasa de éxito durante un largo período de seguimiento, y su longevidad está vinculada a la correcta planificación, elección de materiales y técnicas.

Palabras clave: Rehabilitación oral; Prótesis dental; Aleaciones metalocerámicas.

\section{Introdução}

As próteses parciais fixas metalocerâmicas (PPF) têm sido um substituto para a falta de elementos dentários há muitos anos na odontologia e são uma opção restauradora para dentes vitais ou tratados endodonticamente (Nejatidanesh et al. 2016; 
Lu et al. 2009). Esse sistema é composto por uma infraestrutura metálica que garante a resistência da prótese e uma cobertura de cerâmica para reestabelecer a estética (Güncü et al. 2016). As PPF são consideradas a escolha mais comum entre os profissionais (Güncü et al. 2016), principalmente para as regiões posteriores da mandíbula, devido ao conhecimento de suas propriedades como alta resistência à fratura, longevidade, versatilidade, e relativa simplicidade (Nayakar et al. 2012). As PPF também são bastante utilizadas em dentes anteriores que requer um planejamento criterioso para evitar a exposição de uma cinta metálica na região cervical dos dentes (Morandi et al. 2007).

Os sistemas totalmente cerâmicos surgiram no mercado com intuito de substituir as metalocerâmicas, no entanto, suas peças extensas são mais propícias a fraturas e não podem ser soldadas, limitando assim suas indicações clínicas (Tartaglia et al. 2011). Esse sistema, quando comparado a longo prazo, apresentam taxas de sucesso significativamente menor que as metalocerâmicas, sendo apresentado uma sobrevida de cinco anos das metalocerâmicas unitárias em 95.7\% a 97.6\% (CADTH, 2016). As taxas de sobrevivência das PPF são relatadas em cinco anos de 94,4\%, em 10 anos de $92 \%$ e em 15 anos de $74 \%$ (Ahmed et al. 2017; Pol et al. 2017; Sailer et al. 2007). Essas altas taxas são um dos principais motivos para a alta escolha das PPF nas realidades clínicas (Güncü et al. 2016).

A longevidade das PPF são vinculadas a uma série de fatores como: planejamento de tratamentos prévios à reabilitação; correto planejamento da infra estrutura metálica; qualidade da liga metálica utilizada; compatibilidade entre a cerâmica e a liga metálica escolhida; compatibilidade do cimento entre a estrutura dental e a infraestrutura metálica; grau de carga funcional exercida sobre as restaurações; manutenção apropriada e a precisão com a qual o técnico em prótese dentária e o dentista trabalham em todos os passos de sua confecção (Scurria et al. 1998). Assim o prognóstico satisfatório está sobre responsabilidade do cirurgião-dentista que deve ter conhecimento da longevidade média dessas próteses e dos materiais mais indicados para sua construção e cimentação (Al Refai et al. 2018).

As ligas metálicas de melhor escolha para a confecção da infraestrutura são as ligas nobres por sua biocompatibilidade, porém devido ao alto custo novas ligas foram desenvolvidas (Reddy et al. 2011; Ristic et al. 2014). As ligas não-nobres possuem um módulo de elasticidade quase duas vezes maior que o das ligas nobres sendo motivo para seu uso, principalmente para próteses parciais fixas longas e cantilever (Mohammadi et al. 2021; Wataha et al. 2004). Estudos demonstraram que melhorias nas ligas de $\mathrm{Ni}-\mathrm{Cr}$ as deixaram mais resistentes à corrosão e, portanto, podem ser alternativas adequadas para uso em próteses dentárias (Viennot et al. 2005; Mülders et al. 1996; Ardlin et al. 2005).

Os agentes de cimentação ideal para cimentar peças odontológicas devem apresentar várias características, incluindo biocompatibilidade, baixa solubilidade em água, adesão, radiopacidade, estética, fácil manuseio e baixo custo; além disso, deve evitar a microinfiltração e resistir a forças durante a função oral (Rosenstiel et al. 1998). Atualmente, nenhum cimento dentário disponível atende a todos os requisitos anteriores (Escribano et al. 2006; Heintze et al. 2010). Entretanto os cimentos de ionômero de vidro mostraram resultados favoráveis por estabelecer, através de ligações iônicas, aderências nas interfaces dente-cimento e cimento-metal (Hill et al. 2011).

O cimento ionomérico modificado por resina surgiu com o objetivo de diminuir a sensibilidade à umidade e melhorar as propriedades físicas do ionômero convencional (Amaral et al. 2006). Esse cimento possui resistência tensional diametral e compressiva superiores aos cimentos de fosfato de zinco, policarboxilato e alguns ionômeros convencionais, porém menor que os cimentos totalmente resinosos (BOTTINO et al. 2002). Entretanto, foi comprovado que alguns cimentos a base de ionômero de vidro apresentaram aumento de resistência à compressão depois de dois meses de envelhecimento, assim, podem ser a primeira escolha de cimento quando pensado em custo-benefício (Corrêa et al. 2006).

Desta maneira, este trabalho tem como objetivo enfatizar a sobrevida das metalocerâmicas, através de um caso clínico de reabilitação, utilizando liga metálica $\mathrm{Ni}$-Cr e cimento de ionômero de vidro modificado por resina com acompanhamento de 12 anos. 


\section{Metodologia}

O caso clínico foi submetido e aprovado pelo Comitê de Ética em Pesquisa da Universidade Federal de Mato Grosso do Sul (CEP/UFMS) o protocolo CAAE 39060420.9.0000.0021, sendo este realizado na Faculdade de Odontologia da Universidade Federal de Mato Grosso do Sul (FAODO/UFMS).

\section{Relato de Caso}

Paciente leucoderma do sexo feminino, 56 anos, compareceu à clínica de Prótese Dentária da Faculdade de Odontologia da Universidade Federal de Mato Grosso do Sul, queixando-se da estética de seu sorriso e desejando reabilitar a perda de vários elementos dentários. No exame clínico e pelo exame de imagem (radiografia panorâmica) foi possível observar a falta dos elementos dentários $(14,16,18,25,26,35,36,38$ e 46) e tratamento reabilitador prévio com prótese fixa nos elementos $(11,12,21,24,44$ e 47) (Figura 1 e 2).

Figura 1: Exame clínico inicial. A- vista fontal, B- vista lateral direita, C- vista lateral esquerda.

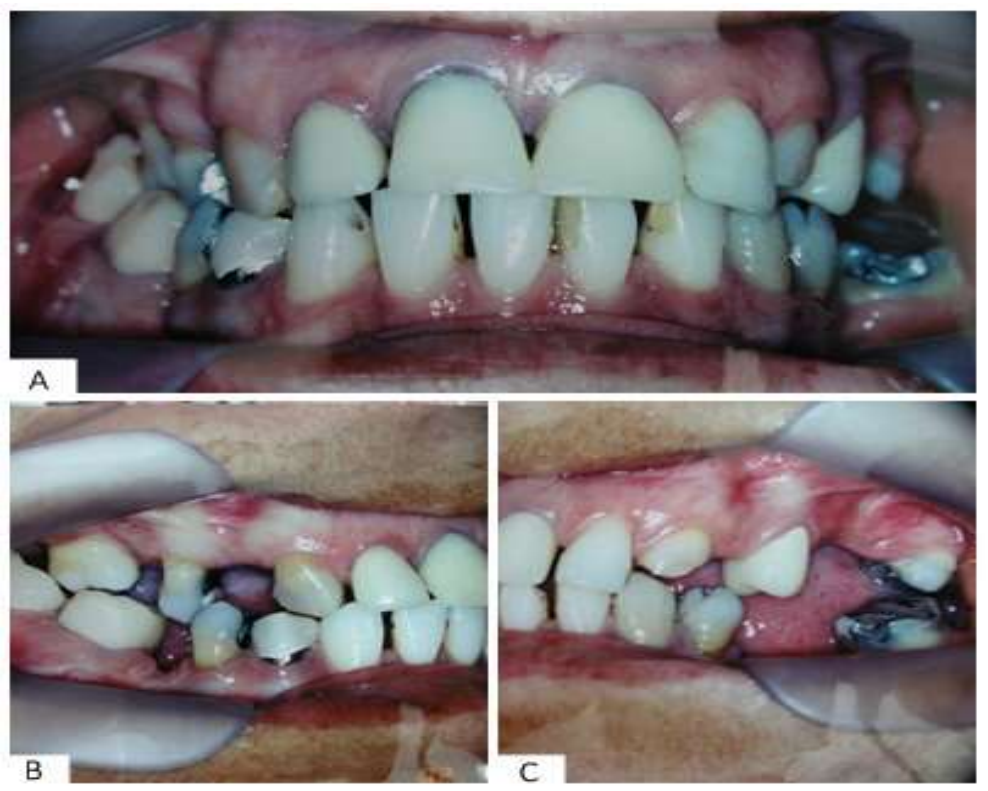

Fonte: Autores.

Figura 2: Exame radiográfico panorâmico inicial

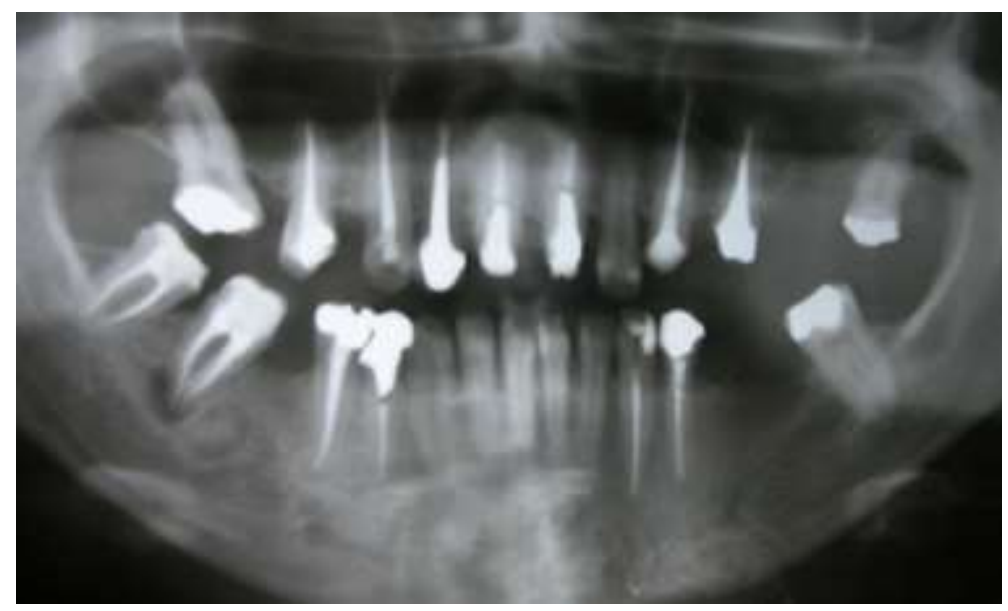

Fonte: Autores. 
Após estudo e análise do caso, foi passado para a paciente vários planejamentos que resultavam em reabilitações com prótese sobre implante ou próteses removíveis, porém sua escolha foi em próteses fixas dento suportadas. Então, foi elaborado uma sequência de tratamento para melhor resolutividade do caso.

Primeiro, foi realizado os tratamentos e retratamentos intraradiculares necessários, assim removendo todas as próteses fixas instaladas, fazendo o preparo do remanescente dentários para colocação de coroas provisórias. Após esta adequação do meio a paciente recebeu tratamento ortodôntico durante seis meses para alinhamento dos dentes no arco e para melhorar o espaço protético que será reabilitado (Figura 3), também foi determinante para posicionar o elemento 24 no espaço dentário do elemento 25 , como estratégia de reabilitação e suporte de forças.

Figura 3: Tratamento Ortodôntico. A- vista fontal, B- vista arcada superior, C- vista arcada inferior.
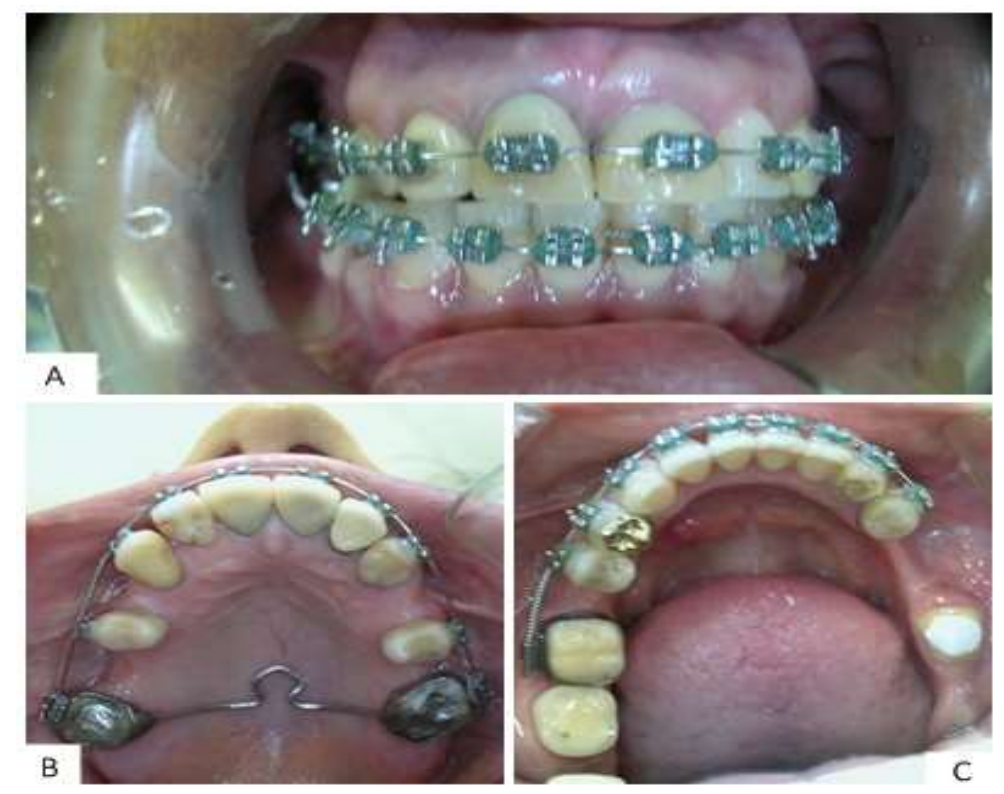

Fonte: Autores.

Foi realizado o enceramento de diagnóstico com modelos montados em articulador, reestabelecendo os planos oclusais e determinando tamanho e forma dos dentes (Figura 4). Nesta etapa foi verificado a necessidade de maior desgaste dental na face vestibular dos dentes anteriores superiores para maior verticalização, melhorando a estética. Assim, foi determinado no arco superior duas próteses parciais fixas (PPF) posteriores de cinco elementos com dois pônticos tanto para o lado esquerdo como direito, e quatro próteses fixas unitárias anteriores. Para o arco inferior foi determinado duas PPF posteriores de cinco elementos com dois pônticos tanto para o lado esquerdo como direito, em vista da exodontia do elemento 47. 
Figura 4: Enceramento de diagnóstico. A- vista fontal, B- vista lateral direita, C- vista lateral esquerda.

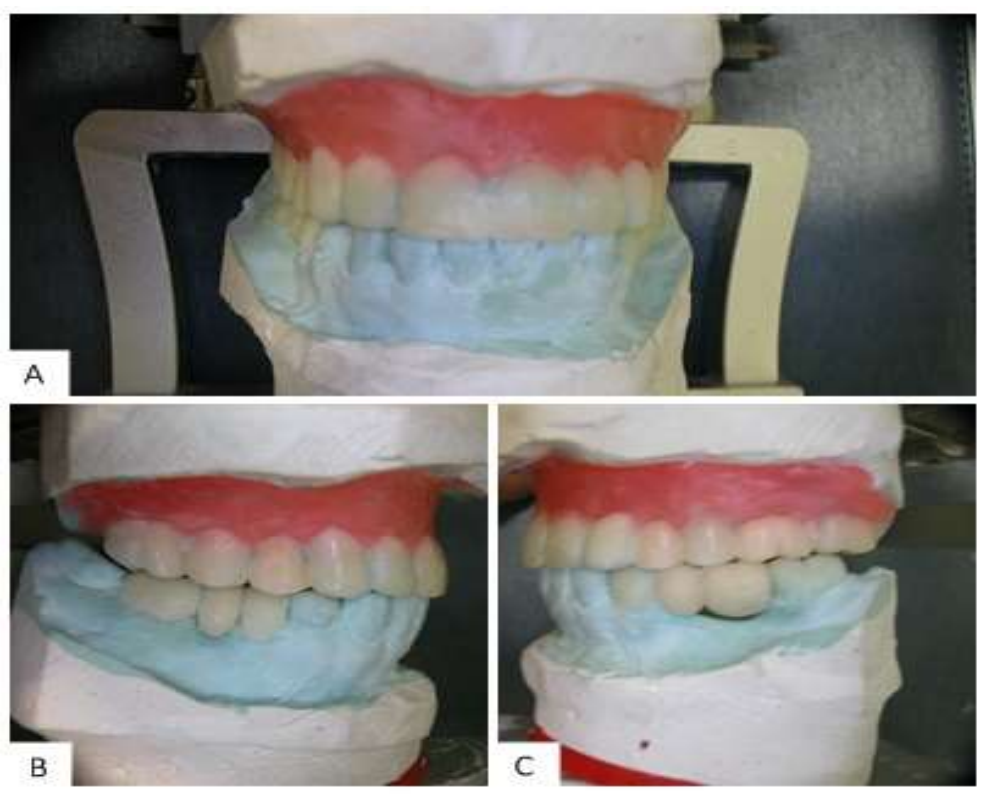

Fonte: Autores.

A infraestrutura metálica foi confeccionada com ligas de Ni-Cr (Figura 5). Sobre a infraestrutura foi aplicado a cerâmica do tipo feldspática, onde nas coroas unitárias se estendeu até o término do coping da face vestibular para evitar exposição de cinta metálica, sendo também aplicado um maior volume na região cervical para melhor assentamento labial, em vista do acentuado desgaste dental feito para verticalização desses elementos (Figura 6).

Figura 5: Infraestrutura metálica CoCr. A- vista arcada superior, B- vista arcada inferior.
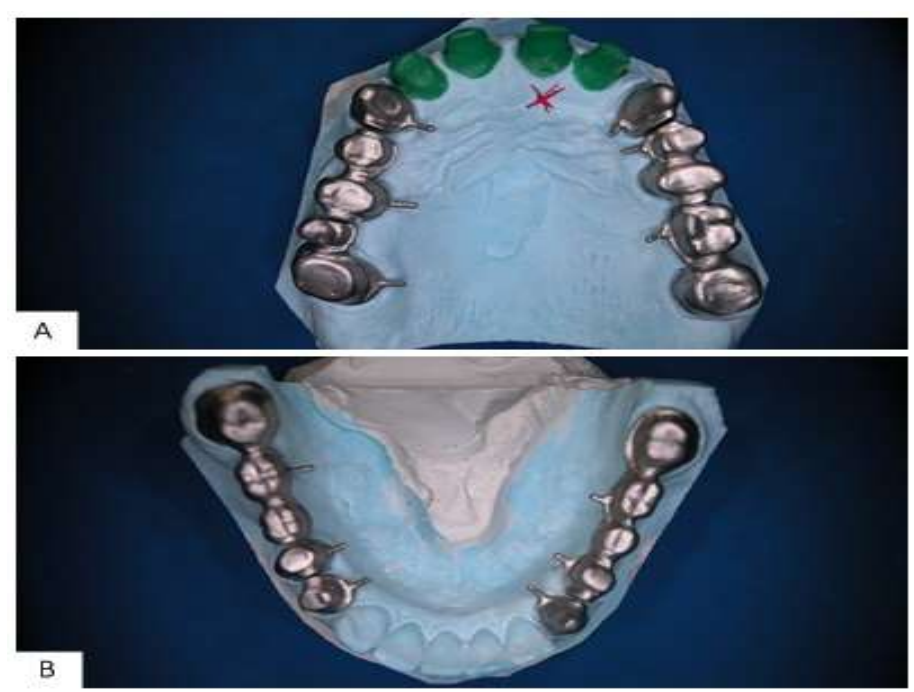

Fonte: Autores. 
Figura 6: Aspecto final após aplicação de cerâmica feldspática. A- vista arcada superior, B- arcada inferior, C- extensão da cerâmica até o termino cervical e aspecto volumoso em área cervical.

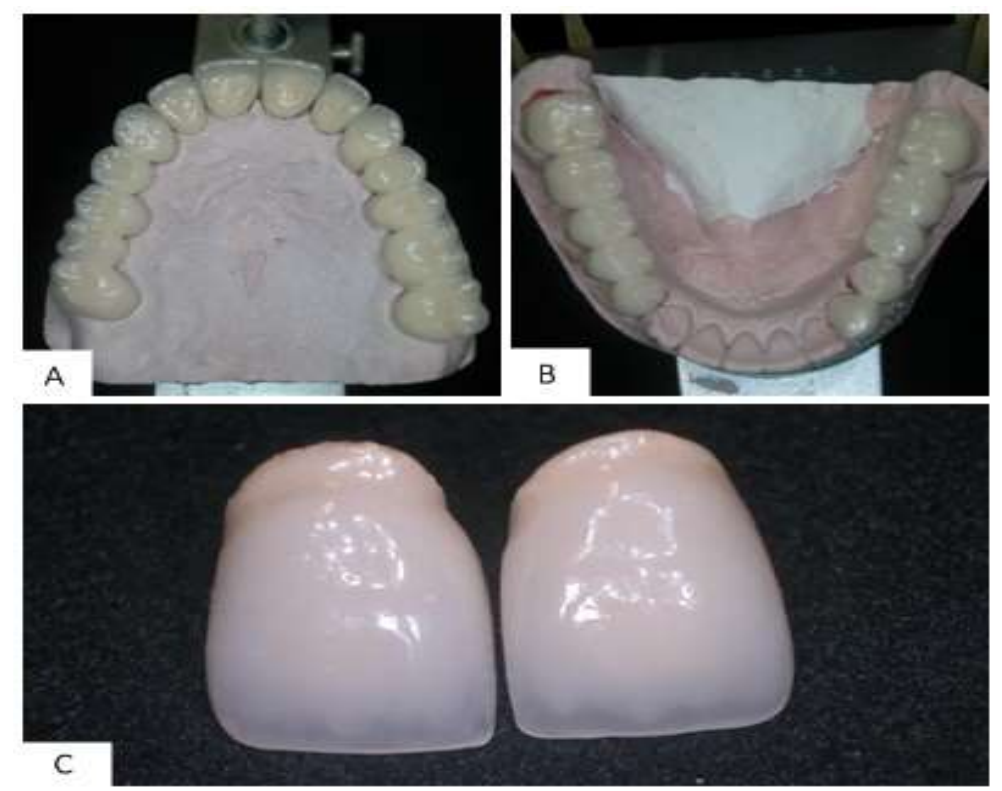

Fonte: Autores.

As próteses metalocêramicas foram cimentadas com o cimento ionômero de vidro modificado por resina Fuji plus C $\left(\mathrm{GC}^{\circledR}\right)$, o qual foi aplicado nas superfícies axiais internas das coroas. As peças eram assentadas com pressão digital, firme e uniforme durante 1 minuto. Ao mesmo tempo, foram aplicados feixes de luz de 1 a 2 segundos do aparelho fotopolimerizador (D700 - Dabi Atlante, potência de saída: $>450 \mathrm{nW} / \mathrm{cm}^{2}$, Ribeirão Preto, SP - Brasil), para então remover os excessos de cimento com sonda exploradora e fio dental. Prosseguiu-se com a complementação da fotopolimerização, expondo por 40 segundos cada face de cada dente pilar com luz do fotopolimerizador.

A Figura 7 mostra o aspecto final da instalação das próteses. A paciente recebeu acompanhamento periódicos, constatando a perfeita função das PPF.

Figura 7: Aspecto final após cimentação. A- vista fontal, B- vista lateral direita, C- vista lateral esquerda.

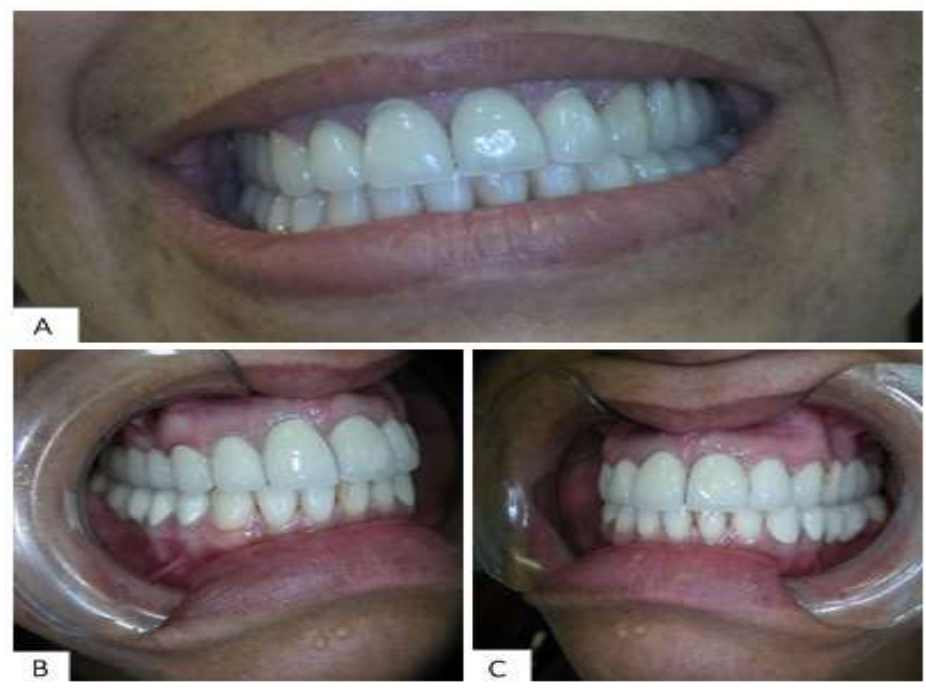

Fonte: Autores. 
Através de exame radiográfico panorâmico foram registrados aspectos após seis anos e 12 anos da instalação das PPF (Figura 8). O acompanhamento pelos exames de imagem e clínicos pode constatar o total sucesso da reabilitação, não sendo verificado desadaptações, infiltração, fraturas das cerâmicas, manchamento gengival indicando degradação metálica, e também sem queixas pela paciente.

Figura 8: Acompanhamento por exame radiográfico. A- Após seis anos da instalação, B- Após 12 anos da instalação.

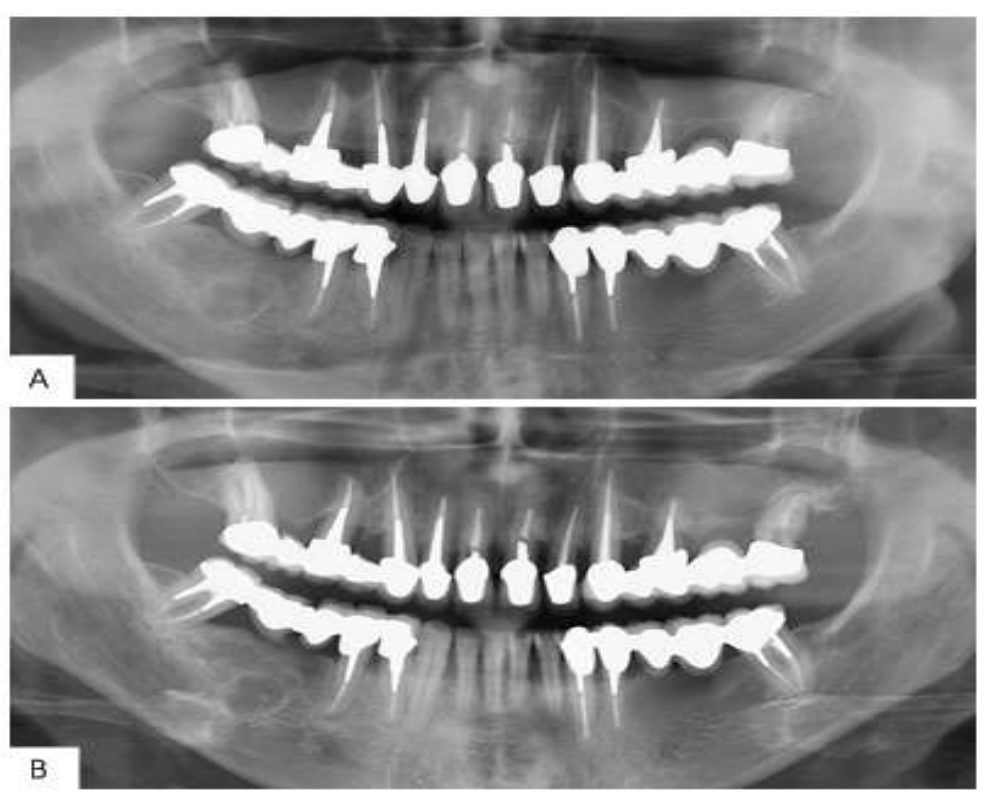

Fonte: Autores.

\section{Discussão}

Clinicamente, a cerâmica usada como material restaurador odontológico têm propriedades como resistência ao desgaste, compatibilidade biológica e potencial estético que conferem grande versatilidade a essas restaurações (Morandi et al. 2007). O uso da infraestrutura metálica sob esse material é o método mais largamente utilizado para compensar sua natureza frágil (Cruickshanks-Boyd, 1981). Assim, o sucesso do conjunto metalocerâmica depende da compreensão dos mecanismos envolvidos nesta união da liga metálica com a cerâmica (Bertolotti et al. 1980). Neste estudo se optou pelo uso de uma liga NiCr por apresentarem capacidade de formar óxidos com um forte potencial de adesão as cerâmicas (Mackert et al. 1984).

Para a grande maioria dos cirurgiões-dentistas, a infraestrutura metálica das coroas afeta de maneira negativa a estética por apresentarem uma cor acinzentada, como no caso da liga Ni-Cr. No entanto, o problema está na espessura da camada opaca, que deve ocultar completamente a cor do metal; uma camada menor propicia uma percepção visual de menor valor na restauração (cor acinzentada), não refletindo a luz recebida em sua superfície na mesma intensidade que os demais dentes (O'Connor et al. 1996). Por isso é necessário fornecer ao técnico o máximo de informações que auxiliem na reprodução das características dentais, principalmente, nas restaurações anteriores como neste estudo que foi de grande importância o planejamento com o técnico, sendo decisivo para a estética ao estender a camada de cerâmica até o término cervical e para o correto assentamento labial ao aplicar maior volume de cerâmica na região cervical.

Tradicionalmente, o cimento de fosfato de zinco tem sido o agente cimentante mais utilizado nas metalocerâmicas, porém apresentam desvantagens bem documentadas, como alta solubilidade que aumenta o risco de infiltração marginal. Apesar desses problemas, esse cimento continua sendo amplamente empregado na clínica odontológica, devido a sua facilidade técnica e sucesso clínico (Bottino et al. 2002). Porém, estudos apontam para o cimento de ionômero de vidro 
modificado por resina como aquele mais promissor quanto à união das melhores características e compensação das desvantagens do fosfato de zinco (Rosenstiel et al. 1998).

A popularidade dos cimentos de ionômero de vidro deve-se à liberação de flúor bem como a sua capacidade de adesão ao esmalte e à dentina. Além disso, com a inclusão de componentes resinosos ao ionômero de vidro, esse material parece exibir desempenho semelhante aos cimentos resinosos quanto à solubilidade, microinfiltração, restando como desvantagem o risco de sorção de água se exposto à umidade precoce (Amaral et al. 2006). Neste caso apresentado foi utilizado cimento de ionômero de vidro modificado por resina.

Segundo Pjeturson et al. (2005), a perda de prótese dentária fixa varia de 0,6\% a 2,7\% após 5 anos devido a complicações biológicas. Neste estudo, com acompanhamento de até 12 anos, não ocorreram falhas biológicas, pois não ocorreu descolamento da prótese por cárie, perda decorrente de doença periodontal ou lasca da porcelana de cobertura, indicando que as estruturas transferiram corretamente as tensões ao longo eixo dos remanescentes dentários e foram capazes de suportar as condições de carregamento existentes. No estudo de Hey et al. (2014), mostraram taxas de sobrevivência de coroas metalocerâmicas superior a 90\% após um período de 6 anos de acompanhamento.

Os autores deste caso clínico desconhecem relatos na literatura que descreve uma extensa reabilitação com prótese fixa metalocerâmica com um acompanhamento de 12 anos, sendo este apresentando um indicativo de taxa de sucesso de 100\%, sendo considerado pela literatura um sucesso excelente (Sailer et al. 2015). Além disso, esse relato se destaca pela abordagem interdisciplinar e singularidade do tratamento realizado, demonstrando uma opção de tratamento eficaz e de menor custo ao paciente. Apesar de tais resultados este relato apresenta algumas limitações como a abordagem clínica utilizando apenas um paciente, o não registro de hábitos do paciente que podem ter influenciado nos resultados encontrados, e a não comparação entre diferentes materiais cimentantes. Os autores propõem que ensaios de casos clínicos randomizados com períodos de acompanhamento maior que 10 anos sejam realizados para melhor entendimento das taxas de sucesso das próteses fixas metalocerâmicas utilizando cimento de ionômero de vidro modificado com resina.

\section{Considerações Finais}

Este trabalho resultou em um relato de caso de uma reabilitação com prótese parcial fixa metalocerâmica com alta taxa de sucesso há longo período de acompanhamento.

Considerando que a longevidade dessas restaurações se encontra vinculada a escolha dos materiais e técnicas utilizadas, os cirurgiões-dentistas devem estar atentos para um correto planejamento das diversas etapas frente aos diferentes casos clínicos.

\section{Referências}

Ahmed, K. E., Li, K. Y., \& Murray, C.A. (2017). Longevity of fiber-reinforced composite fixed partial dentures (FRC FPD)-Systematic review. J Dent. 61:111. https://doi.org/10.1016/j.jdent.2016.08.007

Al Refai, R., \& Saker, S. (2018). Clinical and Radiographic Assessment of Reasons for Replacement of Metal- Ceramic Fixed Dental Prostheses in Patients Referring to Dental School. J Clin Exp Dent. 10(1):e75-e80. https://doi.org/10.4317/jced.53850.

Amaral, R., Ozcan, M., Bottino, M. A., \& Valandro, L. F. (2006) Microtensile bond strength of a resin cement to glass infiltrated zirconia-reinforced ceramic: the effect of surface conditioning. Dent Mater. 22(3):283-90. https://doi.org/10.1016/j.dental.2005.04.021.

Ardlin, B. I., Dahl, J. E., \& Tibballs J. (2005). Static immersion and irritation tests of dental metal-ceramic alloys. Eur J Oral Sci. 113(1):83-9. https://doi.org/10.1111/j.1600-0722.2005.00187.x.

Bertolotti, R. L., \& Moffa, J. P. (1980). Creep rate of porcelain bonding alloys as a function of temperature. Journal of Dental Research, 59, $2062-2065$.

Bottino, M. A., Quintas, A. F. Miyashita, E., Giannini, V. et al. (2002). Estética em reabilitação oral: Metal free. Artes Médicas.

CADTH. (2016). Porcelain-fused-to-metal crowns versus all-ceramic crowns: a review of the clinical cost-effectiveness. CADTH Rapid re-sponse report: summary with crit-ical appraisal. 
Corrêa, L. G. P., \& Ogasawara, T., (2006). Estudos Comparativos de Alguns Cimentos Ionoméricos Convencionais. Revista Matéria, 11, $297-305$.

Cruickshanks-Boyd, D. W. (1981). Alternatives to gold porcelain bonding alloys. Dental Update, 8, 111-119.

Escribano, N., \& de la Macorra, J. C. (2006). Microtensile bond strength of self-adhesive luting cements to ceramic. J Adhes Dent. 8(5):337-41.

Güncü, M. B., Cakan, U., Aktas, G., Güncü, G. N., \& Canay, Ş. (2016) Comparison of implant versus tooth-supported zirconia-based single crowns in a splitmouth design: a 4-year clinical follow-up study. Clin Oral Investig. 20(9):2467-2473. https://doi.org/10.1007/s00784-016-1763-x.

Heintze, S. D. (2010) Crown pull-off test (crown retention test) to evaluate the bonding effectiveness of luting agents. Dent Mater. 26(3):193-206. https://doi.org/10.1016/j.dental.2009.10.004.

Hey, J., Beuer, F., Bensel, T., \& Boeckler, A. F. (2014). Single crowns with CAD/CAM-fabricated copings from titanium: 6-year clinical results. J Prosthet Dent. 112(2):150-4. https://doi.org/10.1016/j.prosdent.2013.09.031.

Hill, E. E., \& Lott, J. (2011). A clinically focused discussion of luting materials. Aust Dent J. 56 Suppl 1:67-76. https://doi.org/10.1111/j.18347819.2010.01297.x.

Lu, Y., Chen, W., Ke, W., \& Wu, S. (2009). Nickel-based (Ni-Cr and Ni-Cr-Be) alloys used in dental restorations may be a potential cause for immunemediated hypersensitivity. Med Hypotheses. 73(5):716-7. https://doi.org/10.1016/j.mehy.2009.04.041.

Mackert, J. R. Jr., Parry, E. E., Hashinger D. T., \& Fairhurst C. W. (1984) Measurement of oxide adherence to PFM alloys. J Dent Res. 63(11):1335-40. https://doi.org/10.1177/00220345840630111701.

Mohammadi, Z., Mahabadi, M., Tabbakhian, G., \& Talaakoob, M. (2021) Bond Strength of Porcelain to Milled Sintered and Casting Base Metal Alloys. J Dent (Shiraz). 2021 Mar;22(1):21-26. https://doi.org/10.30476/DENTJODS.2020.84347.1076.

Morandi, L. B., \& Neto, S. C. B. R., (2007) Reabilitação Oral: Prótese fixa me-talocerâmica anterior inferior com reconstrução de guia. Relato de caso. Arq. bras odontol. 3(1):38-43.

Morandi, L. B., \& Neto, S. C. B. R., (2007). Reabilitação Oral: Prótese fixa me-talocerâmica anterior inferior com reconstrução de guia. Relato de caso. Arq. Bras. Odontol. 3(1):38-43

Mülders, C., Darwish, M., \& Holze, R. (1996) The influence of alloy composition and casting procedure upon the corrosion behaviour of dental alloys: an in vitro study. J Oral Rehabil. 23(12):825-31. https://doi.org/10.1046/j.1365-2842.1996.d01-201.x.

Nayakar, R. P., Patil, N. P., \& Lekha, K. (2012). Comparative evaluation of bond strengths of different core materials with various luting agents used for cast crown restorations. J Indian Prosthodont Soc. 12(3):168-74. https://doi.org/10.1007/s13191-012-0127-8.

Nejatidanesh, F., Moradpoor, H., \& Savabi, O. (2016). Clinical outcomes of zirconia-based implant- and tooth-supported single crowns. Clin Oral Investig. 20(1):169-78. https://doi.org/10.1007/s00784-015-1479-3.

O'Connor R. P., Mackert J. R. Jr., Myers, M. L., \& Parry, E. E. (1996) Castability, opaque masking, and porcelain bonding of 17 porcelain-fused-to-metal alloys. J Prosthet Dent. 75(4):367-74. https://doi.org/10.1016/s0022-3913(96)90027-x.

Pjetursson, B. E., Sailer, I., Makarov, N. A., Zwahlen, M., \& Thoma, D. S. (2015). All-ceramic or metal-ceramic tooth-supported fixed dental prostheses (FDPs)? A systematic review of the survival and complication rates. Part II: Multiple-unit FDPs. Dent Mater. 31(6):624-39. https://doi.org/10.1016/j .dental.2015.02.013.

Pol, C. W. P., Raghoebar, G. M., Kerdijk, W., Boven, G. C., Cune, M. S., \& Meijer, H. J. A. (2018). A systematic review and meta-analysis of 3-unit fixed dental prostheses: Are the results of 2 abutment implants comparable to the results of 2 abutment teeth? J Oral Rehabil. 45(2):147-160. https://doi.org/10.1111/joor.12575.

Reddy, N.R., Abraham, A. P., Murugesan, K., \& Matsa, V. (2011). An in vitro analysis of elemental release and cytotoxicity of recast nickel-chromium dental casting alloys. J Indian Prosthodont Soc. 11(2):106-12. https://doi.org/10.1007/s13191-011-0075-8.

Ristic, L., Vucevic, D., Radovic, L., Djordjevic, S., Nikacevic, M., \& Colic, M. (2014). Corrosive and cytotoxic properties of compact specimens and microparticles of Ni-Cr dental alloy. J Prosthodont. 23(3):221-6. https://doi.org/10.1111/jopr.12100.

Rosenstiel S. F., Land M. F., \& Crispin B. J. (1998). Dental luting agents: A review of the current literature. J Prosthet Dent. 80(3):280-301. https://doi.org/10.1016/s0022-3913(98)70128-3.

Rosenstiel, S. F., Land, M. F., \& Crispin, B. J. (1998) Dental luting agents: A review of the current literature. J Prosthet Dent. 80(3):280-301. https://doi.org/10.1016/s0022-3913(98)70128-3.

Sailer, I., Makarov, N. A., Thoma, D. S., Zwahlen, M., \& Pjetursson, B. E. All-ceramic or metal-ceramic tooth-supported fixed dental prostheses (FDPs)? A systematic review of the survival and complication rates. Part I: Single crowns (SCs). Dent Mater. 2015 Jun;31(6):603-23. https://doi.org/10.1016/j.dental.2015.02.011.

Sailer, I., Pjetursson, B. E., Zwahlen, M., \& Hämmerle, C. H. (2007). A systematic review of the survival and complication rates of all-ceramic and metalceramic reconstructions after an observation period of at least 3 years. Part II: Fixed dental prostheses. Clin Oral Implants Res. 18 Suppl 3:86-96. https://doi.org/10.1111/j.1600-0501.2007.01468.x.

Scurria, M. S., Bader, J. D., \& Shugars, D. A. (1998) Metaanalysis of fixed partial denture survival: prostheses and abutments. J Prosthet Dent. 79:459-64. 
Research, Society and Development, v. 10, n. 8, e26810817218, 2021

(CC BY 4.0) | ISSN 2525-3409 | DOI: http://dx.doi.org/10.33448/rsd-v10i8.17218

Tartaglia, G. M., Sidoti, E., \& Sforza, C. (2011). A 3-year follow-up study of all-ceramic single and multiple crowns performed in a private practice: a prospective case series. Clinics (Sao Paulo). 66(12):2063-70. https://doi.org/10.1590/s1807-59322011001200011.

Viennot, S., Dalard, F., Lissac, M., \& Grosgogeat, B. (2005). Corrosion resistance of cobalt-chromium and palladium-silver alloys used in fixed prosthetic restorations. Eur J Oral Sci. 113(1):90-5. https://doi.org/10.1111/j.1600-0722.2005.00190.x.

Wataha, J. C., \& Messer, R. L. (2004). Casting alloys. Dent Clin North Am. 48(2):vii-viii, 499-512. https://doi.org/10.1016/j.cden.2003.12.010. 